\title{
Reopening Endoscopy after the COVID-19 Outbreak: Indications from a High Incidence Scenario
}

\author{
Luca Ellii ${ }^{1,2}$, Gian Eugenio Tontini ${ }^{1,2}$, Lucia Scaramella ${ }^{1,2}$, Paolo Cantù̀ ${ }^{1,2}$, Matilde Topa ${ }^{1,2}$, Bernardo Dell'Osso ${ }^{3,4}$, Antonio \\ Muscatello $^{2,5}$, Andrea Gori ${ }^{2,5}$, Helmut Neumann ${ }^{6}$, Roberto Penagini ${ }^{1,2}$, Maurizio Vecchi ${ }^{1,2}$
}

\footnotetext{
1) Gastroenterology and Endoscopy Unit, Fondazione IRCCS Ca' Granda Ospedale Maggiore Policlinico, Milan, Italy; 2) Department of Pathophysiology and Transplantation, University of Milano, Milan, Italy; 3) Department of Clinical and Biomedical Sciences, Luigi Sacco University of Milan, Milan, Italy; Aldo Ravelli Center for Neurotechnology and Brain Therapeutics, University of Milan, Milan, Italy. CRC Molecular basis of Neuro-Psycho-Geriatrics diseases, University of Milan, Milan, Italy. 4) Department of Psychiatry and Behavioral Sciences, Stanford University, CA, USA; 5) Infectious Diseases Unit, Department of Internal Medicine, Fondazione IRCCS Ca' Granda Ospedale Maggiore Policlinico, Milan, Italy; 6) Department of Medicine I, University Medical Center Mainz, Mainz, Germany
}

\section{Address for correspondence: Roberto Penagini Gastroenterology and Endoscopy Unit, Fondazione IRCCS Ca' Granda Ospedale Maggiore Policlinico, University of Milano, Milan, Italy \\ roberto.penagini@unimi.it}

Received: 15.06 .2020

Accepted: 25.08.2020
In the latter months of 2019 , a dramatic viral pandemic from coronavirus 2 (SARSCoV-2) causing a severe acute respiratory distress syndrome infection spread rapidly worldwide [1, 2]. Human-tohuman transmission was the main route of infection through the emission of respiratory droplets, but also a fecal-oral route has been recognized as well [1, 3]. Diagnostically, RT-PCR of samples from the respiratory tract $[4,5]$ and thorax CT abnormalities are considered reference standards $[2,6]$. Serological and point-ofcare tests are not yet universally validated but may become a useful diagnostic tool $[4,5]$.

As a response against the spreading of the virus, the majority of countries implemented a lockdown strategy [7]. Healthcare systems and hospitals were reorganized to face and take care of patients with coronavirus disease 2019 (COVID-19) and to contain the spreading of SARSCoV-2 among patients and health personnel [8]. Outpatient services were reduced guaranteeing access only to urgent or undeferrable cases [9]; new strategies were adopted in endoscopy units (EU) in order to reduce the number of investigations carried out [10-13]. Indeed, endoscopy is considered a high-risk activity due to the likely exposure of personnel to patients' possibly infected body fluids $[14,15]$.
These measures effectively slowed down the pandemic, saved lives, and gave time to re-organize healthcare and lifestyles in general. However, until the development of any vaccine or a specific and effective therapy for SARS-CoV-2, a period of "co-habitation" with the virus will be perceived. Once the emergency time has passed, a new "steady state" period of "intermediate viral risk" (the so-called phase 2 ) will be initiated. Our EU located in the city center of Milan, one of the areas most affected at the initiation period of COVID-19, has been recently prepared to restart activity and rebuild the number of investigations while maintaining the maximum level of safety for both patients and personnel.

The aim of this paper is to offer endoscopists a quick reference guide to adapt their endoscopic activity and services after the COVID-19 emergency period and to prepare them for an "intermediate-risk" period.

\section{ENDOSCOPY INDICATIONS, PATIENTS' SELECTION AND TRIAGE}

In the upcoming phase 2 all indications for endoscopic procedures should be maintained, not knowing how long this phase will last $[16,17]$. Particular attention should be addressed for all oncologic indications (e.g., screening and surveillance) to avoid diagnostic delay and the risk of missed cancers among the postponed procedures. Supplementary file 1 summarizes all the indications for non-urgent endoscopy to be adopted in accordance with the current international guidelines [17]. However, a strict triage activity should be continued to properly allocate the endoscopic timing, confirm appropriateness and consequently reduce the risk of SARS-CoV-2 spreading. Roughly, up to $50 \%$ of endoscopic investigations have been reported to be inappropriate, especially in cases of upper endoscopy $[18,19]$.

Every request should be accurately examined by dedicated personnel, preferably via telemedicine or in an outpatient setting [20]. To facilitate the selection of patients "fit for endoscopy" a standardized checklist should be used in order to establish the correct clinical indication and the existence of risk factors for SARS-CoV-2 infection, i.e., the presence of symptoms during the previous 14 days (e.g. fever, cough, dyspnea, ageusia, anosmia) or any contact with SARS-CoV-2 positive subjects. 
Moreover, it is desirable to develop online forms or smartphone applications that will support the optimization of the allocation and time of human resources.

Triage will continue on the day of the procedure, at the EU (Supplementary file 2). The patient's body temperature should be taken, allocating the person to the high-risk category if the temperature, measured with an infrared thermometer (forehead/temporal artery), results $\geq 37.3^{\circ}$ Celsius. Moreover, when available, a rapid point-of-care test for viral RT-PCR and/ or antibodies anti- SARS-CoV-2 will prove useful, although its cost-effectiveness needs to be evaluated. With this respect three strategies are available: 1 ) to test every patient; 2 ) to test every patient in the early phase 2 only, and shift to strategy 3 once epidemiologic data set on reassuring levels; 3 ) to test patients reporting risk factors for SARS-CoV-2 infection only (answer "yes" to items a, b, c in the checklist at Supplementary file 2).

Telemedicine will be a useful tool in phase 2 of the COVID-19 scenario to keep the extent of interpersonal contacts as low as possible. However, telemedicine is not considered to be an official tool for medicine by the law and insurance systems of many countries, and, similarly, the pre-selection of patients using an electronic Case Report Form in order to qualify for access to the endoscopy service (transforming an EU into a "filtered" outpatient facility) should at least be validated by an internal board.

These changes in clinical practice require a parallel change in the legislative, insurance and local protocol systems, bearing in mind that in recent years we have witnessed the rapidly increasing risk of litigation and number of malpractice claims, which have resulted in defensive medicine becoming a wellestablished way of thinking in physicians' decisions [10].

\section{MANAGEMENT OF PATIENTS AND PERSONNEL}

\section{Patients}

All patients should be provided with surgical masks and supplies to perform hand hygiene at the entrance of the EU [21]. In addition, COVID-19 positive and high-risk patients should be provided with a pair of gloves and a disposable light-fabric isolation gown. Accompanying people should wait outside the EU; their access being allowed only for strictly necessary clinical or organizational issues, and with adequate personal protective equipment (PPE). Patients should keep the surgical mask on until the very beginning of oral-route and throughout their anal-route endoscopy examination. For endoscopy, patients should wear a disposable gown and colonoscopy shorts, if available and specific endoscopic masks limiting droplets; all their clothes should be kept in a closed plastic bag. If logistics and time schedule do not allow, low-risk patients undergoing esophagogastroduodenoscopy can wear a disposable light-fabric isolation gown over their clothes. As soon as the endoscopic procedure is finished and before the patient is sent to a dedicated recovery room, a new surgical mask must be worn.

\section{Healthcare Professionals}

In a phase 2 scenario PPE use remains crucial and should not be underestimated, but at the same time it will be reconsidered as a result of the lowering incidence of SARS$\mathrm{CoV}-2$ infection. Clearly, during the endoscopic examination of COVID-19 positive and high-risk patients, in order to protect the medical staff, we suggest high-performance PPE, i.e. a N95 or FFP2/FFP3 respirator, a hairnet, a double pair of gloves, a disposable waterproof surgical gown, a face shield or goggles, and work safety clogs.

In early phase 2 , high-grade PPE is suggested even in the case of low-risk patients' endoscopy. We suggest to wear a disposable light-fabric isolation gown over the waterproof surgical gown, and change it after each endoscopy. Conversely, in an advanced phase 2, where the COVID-19 incidence is hopefully lower and presumably there is greater availability of point-of-care tests, standard endoscopies on low-risk subjects should be considered at a lower risk of infection and, consequently, lighter-grade equipment will be needed, i.e. keeping facial protection (i.e., respirator, hairnet, face shield/ goggles), a single pair of gloves, a disposable light-fabric isolation gown and clogs. In case of PPE shortage, surgical masks may be used as an alternative to respirators.

Particular attention should be paid to more advanced endoscopic techniques such as device-assisted endoscopy, endoscopic ultrasound and endoscopic retrograde cholangiopancreatography. These exams generally last longer than standard endoscopy and require higher personal exposure, thus in this intermediate phase it is recommended to wear high-performance PPE. However, for these procedures we encourage a systematic patients' screening in order to use lighter PPE which are more comfortable for daily routine use during prolonged procedures. Sequences to wear and remove PPE has been previously described [9] and protocols regarding PPE are available at the American Center for Disease Control webpage: https://www.cdc.gov/hai/pdfs/ppe/ppe-sequence.pdf [22].

A filter area should be a room connected with the COVID-19 area. Alternatively, the personnel should consider to initially undress (remove gown and outer pair of gloves) in the COVID-19 endoscopy area and then to complete the remaining steps in the filter area (Fig. 1). The personnel of the low-risk rooms should initially undress (remove light-fabric isolation gown and gloves) in the endoscopic rooms and then carry out the remaining operations out of it. Of course, the outer pair of gloves should be disposed of after each procedure. While treating COVID-19 positive patients, the inner pair of gloves must not be changed between procedures but rather cleaned with hand wash or alcoholic solution.

Preferably, one stable endoscopic team should be scheduled for each room therefore decreasing the basic reproduction number (R0) of any new incident case within the endoscopy facilities. The allocation of personnel to the different endoscopic rooms should consider the risk of a severe outcome in case of COVID-19 against age and/or present co-morbidities; in other words, elderly subjects and/or with co-morbidities should be put in the low-risk rooms. Finally, all non-essential staff should be minimized. It is strictly important to train the personnel periodically on PPE procedures and carry out regular quality controls. Furthermore, it is crucial to test the personnel regularly (twice per month) for RT-PCR and ensure active surveillance in order to prevent viral spread among the endoscopy staff. 


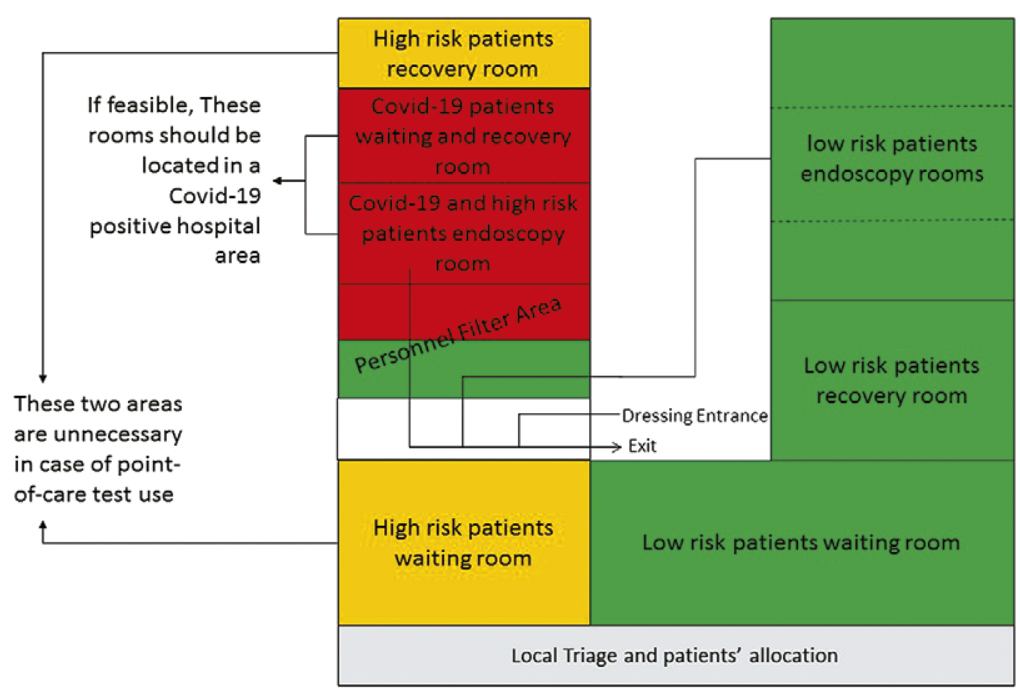

Fig. 1. Example of space management for the endoscopy unit during phase 2.

While preparing to implement the next phase-2 organization of EU, the mental health of the professionals involved needs taking into particular account. Symptoms of burn-out (including mental exhaustion, irritability and insomnia) are expected as consequences of the changed patterns of daily routines [23]. Timely and effective interventions should be made accessible to endoscopists involved in COVID-19 units.

\section{Endoscopic procedures and space management}

During the COVID-19 outbreak, in order to avoid the risk of viral transmission, all EUs reorganized their rooms: generally reserving one room for low-risk patients - oral route, one for low-risk patients - anal route or one room for low-risk patients according to availability, and one for high-risk and COVID-19 positive patients [9]. In the upcoming scenario another adjustment should be applied which is increasing, whenever possible, the number of rooms dedicated to low-risk patients and ideally including a room dedicated to aerosol-generating procedures, considered to be more prone to SARS-CoV-2 infection (i.e., all nasal and oral-route procedures).

Another important organizational issue concerns the storage of single-use devices in the operating rooms, which should not be on shelves, but in protected spaces in order to simplify sanitation.

Furthermore, every effort should be made to maintain one full set of endoscopic armamentaria in a COVID-19 area, when still in place, in order to perform all the endoscopic procedures on COVID-19 inpatients in an isolated environment.

To prevent possible patient-to-patient and patient-topersonnel transmission, several aspects should be systematically taken into account. Overcrowding should be always avoided and an adequate air change per hour should be maintained [24]. When available, negative-pressure endoscopic rooms should then be preferred, especially for COVID-19 patients [25]. As an alternative,well-naturally ventilated rooms may be sufficient especially if an adequate time interval between examinations is set $[15,26,27]$.

Fig. 1 offers a possible layout of the spaces in an EU.

\section{Schedule and duration of procedures}

As stated before [9], patients should enter the EU based on their assessed risk for SARS-CoV-2 infection. Ideally, low-risk patients should be the first procedures to carry out. On the contrary, any endoscopy of COVID-19 positive patients should be the last, with high-risk patients just before them. In the early phase 2 COVID-19 negative inpatients should be allocated in the high-risk group considering the higher prevalence of COVID-19 in the hospital setting, to be then moved to the low-risk group as the pandemic regresses.

Particular attention should be paid to the schedule time of endoscopies: it is advisable to reserve more time to each examination keeping in mind the time spent to adequately wear/don PPE, to the patient's undressing, and other logistic changes such as preventing any overcrowding in the recovery rooms.

\section{Reprocessing of flexible endoscopes and endoscopic} accessories

The standard techniques as described in the American and European guidelines are sufficient and the transmission risk has been reported as low [28]. The healthcare professionals in charge of disinfection should wear high-performance PPE, with a surgical mask being considered adequate $[25,29]$.

\section{Video-capsule endoscopy}

Video-capsule endoscopy (VCE) may be considered a lowrisk procedure, since aerosol is not generally produced but it is well known that patients can cough while swallowing the capsule [30]. In any case, the personnel in attendance should wear adequate PPE, including a facial shield (see previous sections in accordance with the proposed checklist). In the case of VCE for high-risk or COVID-19 positive patients, the investigation should be arranged with precautions similarly to wired endoscopy. When using VCE with a recorder (e.g. PillCam-3) it is suggested to maintain the previous indication of wrapping the recorder and its belt in plastic and secure everything with some tape. Accurate disinfection of the instruments with appropriate products is then recommended. 


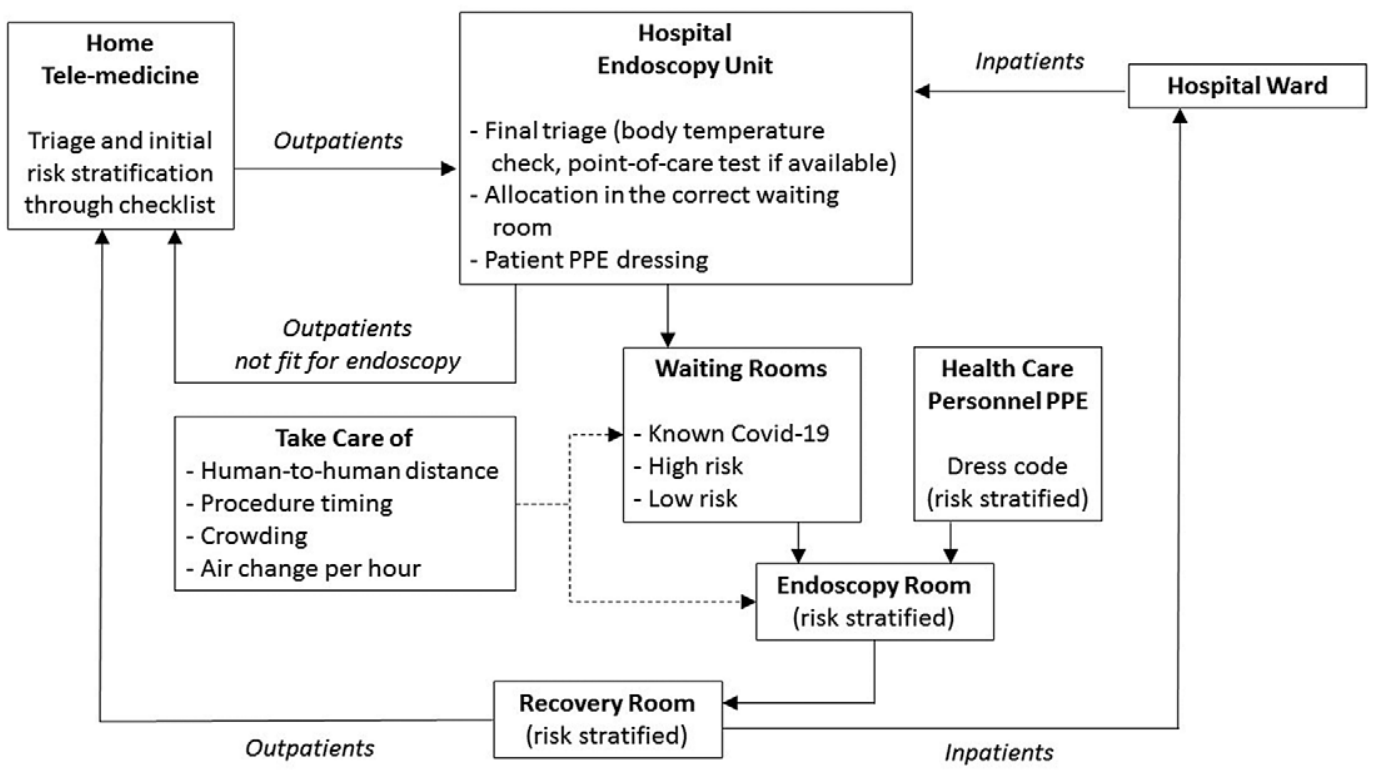

Fig. 2. Endoscopic roadmap of patients during phase 2.

\section{CONCLUSIONS}

With the present manuscript we aimed to support endoscopists in preparing their units for COVID-19 Phase 2, after the severe SARS-CoV-2 pandemic (Fig. 2). We are aware that many factors can influence the above-mentioned indications, especially regarding logistic support, economic status and related resource shortage. Furthermore, we are conscious about the paucity of evidence supporting many of the suggestions we have put forward. However, we think that the experience gained in an EU located in a region where the pandemic levels have been among the highest worldwide, can prove of assistance for other colleagues who find themselves directly on the line at an intermediate epidemic state or, as in our case, after this severe COVID-19 outbreak.

Conflicts of interest: None to declare.

Authors' contributions: L.E., G.E.T, L.S., B.D.O., A.M., A.G: drafted and critically revised the manuscript; B.D.O.: drafted the psychiatric paragraph; M.T.: drafted of the manuscript and updated bibliography; H.N., P.C., M.V., R.P (guarantor): critically revised the manuscript.

Supplementary material: To access the supplementary material visit the online version of the J Gastrointestin Liver Dis at http://dx.doi. org $/ 10.15403 /$ jgld-2687

\section{REFERENCES}

1. Huang C, Wang Y, Li X, et al. Clinical features of patients infected with 2019 novel coronavirus in Wuhan, China. Lancet 2020;395:497-506. doi:10.1016/S0140-6736(20)30183-5

2. Ai T, Yang Z, Hou H, et al. Correlation of Chest CT and RT-PCR Testing in Coronavirus Disease 2019 (COVID-19) in China: A Report of 1014 Cases. Radiology 2020;296:E32-E40. doi:10.1148/ radiol.2020200642
3. Yang Y, Peng F, Wang R, et al. The deadly coronaviruses: The 2003 SARS pandemic and the 2020 novel coronavirus epidemic in China. J Autoimmun 2020;109:102434. doi:10.1016/j.jaut.2020.102434

4. Pan Y, Li X, Yang G, et al. Serological immunochromatographic approach in diagnosis with SARS-CoV-2 infected COVID-19 patients. J Infect 2020;81:e28-e32. doi:10.1016/j.jinf.2020.03.051

5. Infantino M, Damiani A, Gobbi FL, et al. Serological Assays for SARSCoV-2 Infectious Disease: Benefits, Limitations and Perspectives. Isr Med Assoc J 2020;22:203-210.

6. Guan WJ, Ni ZY, Hu Y, et al. Clinical Characteristics of Coronavirus Disease 2019 in China. N Engl J Med 2020;382:1708-1720. doi:10.1056/ NEJMoa2002032

7. Rosenbaum L. Facing Covid-19 in Italy - Ethics, Logistics and Therapeutics on the Epidemic's Front Line. N Engl J Med 2020;382:18731875. doi:10.1056/NEJMp2005492

8. Cook TM, El-Boghdadly K, McGuire B, McNarry AF, Patel A, Higgs A. Consensus guidelines for managing the airway in patients with COVID-19 : Guidelines from the Difficult Airway Society, the Association of Anaesthetists the Intensive Care Society, the Faculty of Intensive Care Medicine and the Royal College of Anaesthetists. Anaesthesia 2020;75:785-799. doi:10.1111/anae.15054

9. Elli L, Rimondi A, Scaramella L, et al. Endoscopy during the Covid-19 outbreak: experience and recommendations from a single center in a high-incidence scenario. Dig Liver Dis 2020;52:606-612. doi:10.1016/j. dld.2020.04.018

10. Elli L, Tenca A, Soncini M, Spinzi G, Buscarini E, Conte D. Defensive medicine practices among gastroenterologists in Lombardy: Between lawsuits and the economic crisis. Dig Liver Dis 2013;45:469-473. doi:10.1016/j.dld.2013.01.004

11. Repici A, Maselli R, Colombo M, et al. Coronavirus (COVID-19) outbreak: what the department of endoscopy should know. Gastrointest Endosc 2020;1;92:192-197. doi:10.1016/j.gie.2020.03.019

12. Branch Infection Control, Health Protection. Infection Control Branch Recommended Personal Protective Equipment (PPE) in hospitals/ clinics under Serious/Emergency Response Level Recommended Personal Protective Equipment for routine patient care and performing aerosol-generating procedures in hospitals/clinics under Serious/ 
Emergency Response Level. 20202 March. Available at: https://www. chp.gov.hk/files/pdf/recommended_ppe_for_nid_eng.pdf

13. Elli L, Tontini GE, Filippi E, et al. Efficacy of endoscopic triage during the Covid-19 outbreak and infective risk. Eur J Gastroenterol Hepatol 2020 Aug 7. doi:10.1097/MEG.0000000000001856

14. Johnston ER, Habib-Bein N, Dueker JM, et al. Risk of bacterial exposure to the endoscopist's face during endoscopy. Gastrointest Endosc 2019;89:818-824. doi:10.1016/j.gie.2018.10.034

15. Tang JW, Li Y, Eames I, Chan PKS, Ridgway GL. Factors involved in the aerosol transmission of infection and control of ventilation in healthcare premises. J Hosp Infect 2006;64:100-114. doi:10.1016/j.jhin.2006.05.022

16. Seda G, Parrish JS. Augmenting Critical Care Capacity in a Disaster. Crit Care Clin 2019;35:563-573. doi:10.1016/j.ccc.2019.06.007

17. ASGE Standards of Practice Committee, Early DS, Ben-Menachem T, et al. Appropriate use of GI endoscopy. Gastrointest Endosc 2012;75:11271131. doi:10.1016/j.gie.2012.01.011

18. Morini S, Hassan C, Meucci G, Toldi A, Zullo A, Minoli G. Diagnostic yield of open access colonoscopy according to appropriateness. Gastrointest Endosc 2001;54:175-179. doi:10.1067/mge.2001.116565

19. Zullo A, Manta R, De Francesco V, Fiorini G, Hassan C, Vaira D. Diagnostic yield of upper endoscopy according to appropriateness: A systematic review. Dig Liver Dis 2019;51:335-339. doi:10.1016/j. dld.2018.11.029

20. Rolston DM, Meltzer JS. Telemedicine in the intensive care unit: its role in emergencies and disaster management. Crit Care Clin 2015;31:239255. doi:10.1016/j.ccc.2014.12.004

21. Federal Register, Volume 75, Issue 119 (Tuesday, June 22, 2010). Rules and Regulations. Available at:https://www.govinfo.gov/content/pkg/ FR-2010-06-22/html/2010-13947.htm

22. World Health Organization. Rational use of personal protective equipment for coronavirus disease (COVID-19). Interim guidance. 27
February 2020. World Health Organization: https://apps.who.int/iris/ handle/10665/331215

23. Fiorillo A, Gorwood P. The consequences of the COVID-19 pandemic on mental health and implications for clinical practice. Eur Psychiatry 2020;63:e32. doi:10.1192/j.eurpsy.2020.35

24. Atkinson J, Chartier Y, Pessoa-Silva CL, Jensen P, Li Y, Seto WH. Editors. Natural Ventilation for Infection Control in Health-Care Settings. WHO Guidelines 2009. Available at: https://www.who.int/ water_sanitation_health/publications/natural_ventilation/en/

25. ASGE Quality Assurance in Endoscopy Committee, Calderwood AH, Day LW, et al. ASGE guideline for infection control during GI endoscopy. Gastrointest Endosc 2018;87:1167-1179. doi:10.1016/j. gie.2017.12.009

26. Escombe AR, Oeser CC, Gilman RH, et al. Natural ventilation for the prevention of airborne contagion. PLoS Med 2007;4:e68. doi:10.1371/ journal.pmed.0040068

27. Escombe AR, Ticona E, Chávez-Pérez V, Espinoza M, Moore DAJ. Improving natural ventilation in hospital waiting and consulting rooms to reduce nosocomial tuberculosis transmission risk in a low resource setting. BMC Infect Dis 2019;19:88. doi:10.1186/s12879-019-3717-9

28. Repici A, Aragona G, Cengia G, et al. Low risk of covid-19 transmission in GI endoscopy. Gut 2020 Apr 22. doi:10.1136/gutjnl-2020-321341

29. Beilenhoff U, Biering H, Blum R, et al. Reprocessing of flexible endoscopes and endoscopic accessories used in gastrointestinal endoscopy: Position Statement of the European Society of Gastrointestinal Endoscopy (ESGE) and European Society of Gastroenterology Nurses and Associates (ESGENA) - Update 2018. Endoscopy 2018;50:1205-1234. doi:10.1055/a-0759-1629

30. Tontini GE, Manfredi G, Orlando S, et al. Endoscopic ultrasonography and small-bowel endoscopy: Present and future. Dig Endosc 2019;31:627-643. doi:10.1111/den.13429 